\title{
A COMMON FIXED POINT THEOREM IN A CONE METRIC SPACE
}

\author{
K. Jha \\ Department of Natural Science (Mathematics), \\ School of Science, Kathmandu University \\ P.O. BOX NO. 6250, Kathmandu, NEPAL. \\ E-mail: jhaknh@yahoo.co.in; jhakn@ku.edu.np \\ Received 1 August, 2008; Revised 23 November, 2008
}

\begin{abstract}
A common fixed point theorem for a pair of weakly compatible mappings is proved in a cone metric space which extends and improves various well-known similar results.
\end{abstract}

Key words and phrases: Common fixed point, weakly compatible maps, cone metric space.

2000 Mathematics Subject Classification: 54 H 25, 47 H 10.

\section{INTRODUCTION}

The study of common fixed points of mappings satisfying certain contractive conditions has been at the center of vigorous research activity. In 1976, Jungck [3] proved a common fixed point theorem for commuting mappings, generalizing the famous Banach contraction principle. Sessa [8] introduced the notion of weakly commuting maps. Also, Jungck [4] introduced the notion of compatible mappings in order to generalize the concept of weak commutativity. Again, Pant [6] defined $R$-weakly commutating maps and established some common fixed point theorems, assuming the continuity of at least one of the mappings. Kannan [9] proved the existence of fixed point for a mapping that can have a discontinuity in a domain, however maps involved in each case were continuous at the fixed point.

Jungck and Rhoades [5] defined a pair of self mappings to be weakly compatible if they commute at their coincidence points. Then, applying these concepts, several authors have obtained coincidence point results for various classes of mappings in a metric space. On the other hand, Huang and Zhang [2] generalized the concept of a metric space, replacing the set of real numbers by an ordered Banach space and obtained some fixed point theorems for mappings satisfying different contractive conditions. Recently, Abbas and Jungck [1] proved some common fixed point theorems for weakly compatible mappings in the setting of a cone metric space.

The aim of this paper is to establish a common fixed point theorem for a pair of weakly compatible mappings in a cone metric space, without exploiting the notion of the 
continuity. This paper extends and improves various well-known similar results. The following notions have been used to prove the main result.

Let $E$ be a real Banach space. A subset $P$ of $E$ is called a cone if, and only if

(a) $P$ is closed, nonempty and $P \neq\{0\}$;

(b) $a, b \in \Re, a, b \geq 0$, and $x, y \in P$ implies $a x+b y \in P$;

(c) $P \cap(-P)=\{0\}$.

Given a cone $P \subset E$, we define a partial ordering $\leq$ with respect to $P$ by $x \leq y$ if, and only if $y-x \in P$. A cone $P$ is called normal if there is a number $K>0$ such that for all $x, y \in E$, the inequality $0 \leq x \leq y$ implies $\|x\| \leq K\|y\|$.

The least positive number satisfying the above inequality is called the normal constant of $P$, while $x \ll y$ stands for $y-x \in \operatorname{int} P$ (interior of $P$ ).

DEFINITION 1.1.[2] Let $X$ be a non empty set. Suppose that the mapping $d$ : $X \times X \rightarrow E$ satisfies

$(d 1) 0 \leq d(x, y)$ for all $x, y \in X$ and $d(x, y)=0$ if, and only if $x=y$;

$(d 2) d(x, y)=d(y, x)$ for all $x, y \in X$;

$(d 3) d(x, y) \leq d(x, z)+d(z, y)$ for all $x, y, z \in X$.

Then $d$ is called a cone metric on $X$ and $(X, d)$ is called a cone metric space. The concept of a cone metric space is more general than that of a metric space.

DEFINITION 1.2. Let $(X, d)$ be a cone metric space. We say that $\left\{x_{n}\right\}$ is

(e) a Cauchy sequence if for every $c$ in $E$ with $c \gg 0$, there is $N$ such that for all $n, m>N, d\left(x_{n}, x_{m}\right) \ll c$;

(f) a convergent sequence if for every $c$ in $E$ with $c \gg 0$, there is $N$ such that for all $n>N, d\left(x_{n}, x\right) \ll c$ for some fixed $x$ in $X$.

A cone metric space $X$ is said to be complete if every Cauchy sequence in $X$ is convergent in $X$.

It is known that $\left\{x_{n}\right\}$ converges to $x \in X$ if, and only if $d\left(x_{n}, x\right) \rightarrow 0$ as $n \rightarrow \infty$. Also, the limit of a convergent sequence is unique provided $P$ is a normal cone with normal constant $K$ (refer Huang and Zhang [2]).

DEFINITION 1.3. Let $f$ and $g$ be self mappings of a set $X$. If $w=f x=g x$ for some $x$ in $X$, then $x$ is called a coincidence point of $f$ and $g$, and $w$ is called a point of coincidence of $f$ and $g$.

DEFINITION 1.4.([5]) Two self mappings $f$ and $g$ of a set $X$ are said to be weakly compatible if they commute at their coincidence points; that is, if $f u=g u$ for some $u \in X$, then $f g u=g f u$. 
PROPOSITION 1.5.([1]) Let $f$ and $g$ be weakly compatible self mappings of a set $X$. If $f$ and $g$ have a unique point of coincidence, that is, $w=f x=g x$, then $w$ is the unique common fixed point of $f$ and $g$.

\section{MAIN RESULT}

In this section, a common fixed point theorem is proved for a pair of self mappings defined on a cone metric space under a plane contractive condition.

THEOREM Let $(X, d)$ be a cone metric space, and $P$ be a normal cone with normal constant $K$. Suppose that the mappings $f, g: X \rightarrow X$ satisfy the contractive condition

$$
d(f x, f y) \leq r[d(f x, g y)+d(f y, g x)+d(f x, g x)+d(f y, g y)]
$$

where $r \in[0,1 / 4)$ is a constant. If the range of $g$ contains the range of $f$ and $g(X)$ is complete subspace of $X$, then $f$ and $g$ have a unique coincidence point in $X$. Moreover, if $f$ and $g$ are weakly compatible, then $f$ and $g$ have a unique common fixed point.

PROOF: Let $x_{0}$ be an arbitrary point in $X$. Then, since $f X \subset g X$, we choose a point $x_{1}$ in $X$ such that $f\left(x_{0}\right)=g\left(x_{1}\right)$. Continuing this process, having chosen $x_{n}$ in $X$, we obtain $x_{n+1}$ in $X$ such that $f\left(x_{n}\right)=g\left(x_{n+1}\right)$. Then,

$d\left(g x_{n+1}, g x_{n}\right)=d\left(f x_{n}, f x_{n-1}\right)$

$$
\begin{aligned}
& \leq r\left[d\left(f x_{n}, g x_{n-1}\right)+d\left(f x_{n-1}, g x_{n}\right)+d\left(f x_{n}, g x_{n}\right)+d\left(f x_{n-1}, g x_{n-1}\right)\right] \\
& \leq 2 r\left[d\left(g x_{n+1}, g x_{n}\right)+d\left(g x_{n}, g x_{n-1}\right)\right] .
\end{aligned}
$$

So, we have

$$
d\left(g x_{n+1}, g x_{n}\right) \leq h d\left(g x_{n}, g x_{n-1}\right), \text { with } h=\frac{2 r}{1-2 r} .
$$

Now, for $n>m$, we get

$$
\begin{aligned}
d\left(g x_{n}, g x_{m}\right) & \leq d\left(g x_{n}, g x_{n-1}\right)+d\left(g x_{n-1}, g x_{n-2}\right)+\ldots+d\left(g x_{m+1}, g x_{m}\right) \\
& \leq\left(h^{n-1}+h^{n-2}+\ldots+h^{m}\right) d\left(g x_{1}, g x_{0}\right) \\
& \leq \frac{h^{m}}{(1-h)} d\left(g x_{1}, g x_{0}\right),
\end{aligned}
$$

which, using the normality of cone $P$, implies that

$$
\left\|d\left(g x_{n}, g x_{m}\right)\right\| \leq \frac{h^{m}}{(1-h)} K\left\|d\left(g x_{1}, g x_{0}\right)\right\| .
$$

Then, $d\left(g x_{n}, g x_{m}\right) \rightarrow 0$ as $n, m \rightarrow \infty$, and so $\left\{g x_{n}\right\}$ is a Cauchy sequence in $X$. Since $g(X)$ is a complete subspace of $X$, so there exists $q$ in $g(X)$ such that $g x_{n} \rightarrow q$, as $n \rightarrow \infty$. Consequently, we can find $p$ in $X$ such that $g(p)=q$. Thus, 


$$
\begin{aligned}
d\left(g x_{n}, f p\right) & =d\left(f x_{n-1}, f p\right) \\
& \leq r\left[d\left(f x_{n-1}, g p\right)+d\left(f p, g x_{n-1}\right)+d\left(f x_{n-1}, g x_{n-1}\right)+d(f p, g p)\right],
\end{aligned}
$$

which, using the normality of cone $P$, implies that

$$
\left\|d\left(g x_{n}, f(p)\right)\right\| \leq K r\left\|d\left(g x_{n-1}, g p\right)\right\|=0, \text { as } n \rightarrow \infty .
$$

Hence, $d\left(g x_{n}, f p\right) \rightarrow 0$ as $n \rightarrow \infty$. Also, we have $d\left(g x_{n}, g p\right) \rightarrow 0$ as $n \rightarrow \infty$. The uniqueness of a limit in a cone metric space implies that $f(p)=g(p)$.

Again, we show that $f$ and $g$ have a unique point of coincidence.

For this, if possible, assume that there exists another point $t$ in $X$ such that $f(t)=g(t)$. Then, we have

$$
\begin{aligned}
d(g t, g p) & =d(f t, f p) \\
& \leq r[d(f t, g p)+d(f p, g t)+d(f t, g t)+d(f p, g p)],
\end{aligned}
$$

which, using the normality of cone $P$, implies that $\|d(g t, g p)\|=0$, and so, we have $g t=g p$. Finally, using the Proposition 1.5, we conclude that $f$ and $g$ have a unique common fixed point.

This completes the proof of the theorem.

We now give an example to illustrate the above Theorem 2.1.

EXAMPLE 2.2: Let $E=I^{2}$, for $I=[0,1], P=\{(x, y) \in E: x, y \geq 0\} \subset I^{2}$, $d: I \times I \rightarrow E$ such that $d(x, y)=(|x-y|, \alpha|x-y|)$, where $\alpha>0$ is a constant. Define $f x=\frac{\alpha x}{1+\alpha x}$, for all $x \in I$ and $g x=\alpha x$ for all $x \in I$. Then, for $\alpha=1$, both the mappings $f$ and $g$ are weakly compatible and satisfy all the conditions of the above theorem with $x=0$ as a unique common fixed point.

REMARK 2.3: The above theorem extends the results of Abbas and Jungck [1]. Also, it improves the results of Huang and Zhang [2] which itself is a generalization of result of Kannan [9] and unifies the results of Jungck [3] and Pant [6].

\section{ACKNOWLEDGEMENT}

The author gratefully acknowledges the Abdus Salam School of Mathematical Sciences (ASSMS), GC University, Lahore, Pakistan for providing the Post Doctoral Research Fellowship.

The author also thanks to Prof. J. Pecaric, Mathematics Department, University of Zagreb, Zagreb, Croatia and Prof. G. E. Karadzhov, Institute of Mathematics and Information, Bulgarian Academy of Sciences, Sofia, Bulgaria for their valuable suggestions. 


\section{REFERENCES}

1. ABBAS. M AND JUNGCK.G., Common fixed point results for noncommuting mappings without continuity in cone metric space, J. Math. Anal. Appl., 341(2008), $416-420$.

2. HUANG. L. G. AND ZHANG.X., Cone metric spaces and fixed point theorems of contractive mappings, J. Math. Anal. Appl., 332(2)(2007), 1468 - 1476.

3. JUNGCK. G., Commutative maps and fixed points, Amer. Math. Monthly, 83(1976), 261 - 263.

4. JUNGCK. G., Compatible mappings and common fixed points. Internat. J. Math. Math. Sci., 9(4)(1986), 771 - 779.

5. JUNGCK. G. AND RHOADES. B. E., Fixed point for set valued functions without continuity, Indian J. Pure Appl. Math., 29(3)(1998), 227 - 238.

6. PANT. R. P., Common fixed points of non-commutating mappings, J. Math. Anal. Appl., 188(1994), 436 - 440.

7. PANT. R. P., PANT. V AND JHA. K., A note on common fixed points under strict contractive conditions, J. Math. Anal. Appl., 227(2002), 879 - 880.

8. SESSA, S., On a weak commutativity condition of mappings in fixed point consideration, Publ. Inst. Math. Soc., 32(1982), 149 - 153.

9. KANNAN, R., Some results on fixed points, Bull. Cal. Math. Soc., 60(1968), 71 $-76$. 